\title{
Mechanisms of Nanoparticle Formation by Laser Ablation
}

\author{
Tatiana Itina ${ }^{1}$ and Karine Gouriet ${ }^{2}$ \\ ${ }^{1}$ CNRS/University of Lyon, \\ ${ }^{2}$ CNRS/Ecole des Mines de Saint-Etienne,
}

France

\section{Introduction}

During the last decade, numerous experiments have been performed, clearly demonstrating that an interaction of a short (several nanoseconds or shorter) laser pulse with a solid target leads to the formation of nanoparticles. This technique, known as pulsed laser ablation (PLA), has become a promising method of the synthesis of nanoclusters for photonics, electronics and medicine (Movtchan et al., 1995; Yamada et al., 1996; Makimura et al., 1996; Geohegan et al., 1998; Albert et al., 2003). The PLA method has several advantages compared to more traditional techniques. In particular, it was shown that this method provides a possibility for chemically clean synthesis, which is difficult to achieve under more conventional nanoparticle production conditions. In addition, several experimental studies indicated that the cluster size distribution could be controlled in PLA by carefully choosing the laser irradiation parameters and properties of the background gas. Furthermore, laser ablation allows for an easy production of colloidal metal nanoparticles for biological and medical applications.

Despite the large number of the experimental results, the theoretical understanding of the physical and chemical mechanisms leading to the formation of nanoparticles during the PLA is still lacking. The number of theoretical studies of these mechanisms remains limited because both the continuum hydrodynamic models and the classical nucleation theory become inapplicable under the typical PLA conditions. Under these conditions, laser plume expansion and all collisional processes inside the plume occur so rapidly that equilibrium conditions are not attained. In addition, fast laser energy deposition may induce an explosive volume ejection rather than an equilibrium surface evaporation. Nevertheless, the laser ablation process is often described by a thermal desorption model, which considers the ablation as a rather slow layer-by-layer evaporation of monomers from the target surface (Anisimov, 19968). The presence of nanoparticles in the laser plume is then explained by using a Zel'dovich-Raizer condensation model (Zeldovich, 1966; Luk'yanchuk et al., 1998; Gusarov et al., 2000). This approach is appropriate for interpretation of the experimental findings obtained in laser ablation with hundred of nanoseconds and longer laser pulses, and in the presence of a background gas (Ohkubo et al., 2003; Boldarev et al., 2001). In the PLA with shorter laser pulses, however, clusters can be ejected directly from the target as a result of the target disintegration by laser-induced explosion-like process (Bulgakov, 2004; Amoruso et al., 2004; Zhigilei, 2003). In this case, the common thermal desorption and 
condensation model is insufficient and only a detailed molecular-level simulation can provide a complete description of the nanoparticle formation process. One of the possible approaches for such simulations is to combine the molecular dynamics technique (MD) with the direct simulation Monte Carlo method (DSMC) (Zhigilei \& Garrison, 2000; Bird, 1994; Itina et al., 2002; Zeifman et al., 2002; Mizuseki et al., 2001; Briehl \& Urbassek, 1999). This combination allows one to properly account for both the processes of cluster ejection and their following evolution during the laser plume expansion as a result of the gas-phase collisions.

So far, only several theoretical models were proposed to explain the growth of nanoparticles. For instance, such models as the model of Rice Ramsperger and Kassel (RRK) (Malakhovskii \& Ben-Zion, 2002) and the Classical Nucleation Theory (CNT) were used in many studies (Kinjo et al. 1999; Zhong et al., 2006; Schenter et al., 1999). An alternative approach that can be successfully used for the investigation of the condensation-evaporation processes in small systems is based on the molecular dynamics (MD) method. In this method, molecular movement and interactions are simulated directly and one can easily study system dynamics (Kinjo et al. 1999; Zhong et al., 2006; Frenkel \& Smit, 1996). Therefore, MD was also used to study the formation and evolution processes of clusters of many materials, and the advantages of this approach were demonstrated (Malakhovskii \& Ben-Zion, 2002; Zhong et al., 2006).

Here, first we study the evolution of clusters in the presence of the same gas species. The study focuses on the influence of the surrounding gas and cluster parameters on the time evolution of clusters and small molecules formed in typical laser ablation experiments. The cluster parameters, such as cluster temperature and size, are chosen based on the cluster properties found in the ablation calculations. In the same way, the corresponding characteristics of the surrounding environment, such as gas density and temperature, are set to represent the ablated plume. Calculations are performed for two different materials, molecular matrix and metal. The roles of both simulation parameters and material properties are investigated. The applicability of the RRK theory is verified.

Then, we present the numerical results obtained with a hybrid MD-DSMC model of PLA for different laser pulses and fluences. The objective of this study is to bridge a gap between the previous molecular dynamics simulations and typical ablation experiments. In fact, the proposed combination of methods allows us to attain both time and length scales typical for ablation experiments. By using the combined model, we investigate the time evolution of clusters in the plume. We examine different types of collisions leading to cluster growth and decay. As a result, we determine the major collisional processes responsible for the observed changes in cluster distributions. These results are related to the recent experimental results on the ablation plume dynamics.

\section{Simulation details}

\subsection{MD calculations of cluster evolution}

In the first part of our calculations, classical molecular dynamics (Frenkel \& Smith, 1996 and Zhigilei et al., 1998) is used to study a system composed by a nickel cluster surrounded by a gas of the same atoms. In many applications, metal nanoparticles are used due to their optical (plasmonic), and chemical (sensing) properties (Amoruso et al., 2007). The effective Embedded Atom Model (EAM) potential (Daw \& Baskes, 1984) is used in these calculations. This force field contains two terms: a pairwise additive interaction $\phi$ of the atomic cores 
shielded at short interatomic distances and secondly a contribution $F(\rho)$ of delocalized electrons to the energy, which is modeled by multi-body term assuming a linear superposition $\rho_{i}$ of atomic electron densities $\rho_{\text {at. }}$ The energy of an atom $i$ can be written as

$$
E_{i}=F_{i}\left[\rho_{i}\right]+\frac{1}{2} \sum_{i \neq j} \phi_{i j}\left(r_{i j}\right)
$$

The subscripts $i$ and $j$ run over the number of atoms $\mathrm{N}$ in the system. A cut-off radius of $0.53 \mathrm{~nm}$ is used for the electron density and the pair potential contribution to the $\mathrm{Ni}-\mathrm{Ni}$ interaction.

First, we form a cluster of $\mathrm{N}$ particles with the initial temperature $\mathrm{T}_{0}$. Then, a background gas is generated based on the corresponding gas number density and temperature. The volume of the cell is constant and equal to $100 \times 100 \times 100 \mathrm{~nm}^{3}$. Periodic boundary conditions in all directions are imposed. After generating the gas, we heat it, so that each gas particles has a velocity based on the Maxwell-Boltzmann distribution with a given gas temperature. Then the cluster is located in the center of the cell. Thus, we can vary four basic parameters: two for the cluster (initial temperature and size) and two for the gas (temperature and density).

In the calculations, we monitor the following processes (i) simple collision

$$
A_{i}+A \stackrel{v_{\text {coll }}}{\longrightarrow} A_{i}+A
$$

with the collision rate $v_{\text {colli }}$ and (ii) evaporation and condensation processes

$$
A_{i}+A \stackrel{v_{e}, v_{c}}{\longleftrightarrow} A_{i+1}
$$

with reaction rates $v_{e}$ and $v_{c}$ respectively. The $v_{e}\left(\right.$ or $\left.v_{c}\right)$ rate is calculated as the ratio of the number of evaporated cluster molecules (or condensated gas molecules on the cluster) with the time step $\delta$ t. This time step is sufficiently larger than the MD calculation time step $(1 \mathrm{fs}$ for nickel) and in order to 50 ps. Calculations typically continue until about 3 ns. This time delay is long enough for the establishment of a dynamic equilibrium between the evaporation and condensation processes. This condition corresponds to $\sim 2 \mathrm{~ns}$ in the present calculations.

To determine the number of cluster molecules, we used the neighbor method. Before starting the simulation, we define differently the both cluster and gas molecules. We calculate the neighbor list at each $25 \mathrm{fs}$, and the cluster molecule list at each 50 ps. If a molecule own the cluster at the last step is not in the new list, this molecule is evaporated and we increased the number of evaporated molecules $\mathrm{N}_{\text {evai }}$ and inversely. To investigate the influence of each parameter on the others, we ran several simulations with 3 fixed parameters.

The cluster evaporation process can be described as unimolecular thermal decomposition process by using the RRK theory (Malakhovskii \& Ben-Zion, 2002 21). In the RRK theory, the evaporation and the condensation of clusters are controlled by the sublimation energy $D_{n}=E_{n}-E_{n-1}$, where $\mathrm{E}_{\mathrm{n}}$ is the absolute binding energy of the cluster with $\mathrm{n}$ molecules. The evaporation rate is defined as following

$$
v_{e}^{R R K}=v_{0} g_{n}\left[\frac{E_{n}-D_{n}}{E_{n}}\right]^{s_{n}-1}
$$


where $v_{0}$ is the typical molecular vibrational frequency, $v_{n}$ is the number of vibrational degrees of freedom ( 1 for dimers and $3 \mathrm{~N}-6$ for larger clusters), $\mathrm{g}_{\mathrm{n}}$ is the number of surface molecules and $D_{n}$ is the molecular evaporation energy. For clusters larger than 50 molecules in size, the evaporation rate can be approximate by

$$
v_{e}^{R R K}=v_{0} g_{n} \exp \left[-\frac{D_{n}}{E_{n}}\right]
$$

For large clusters, we use the following approximations (Malakhovskii \& Ben-Zion, 2002)

$$
g_{n}=(36 \pi)^{1 / 3}\left(N^{1 / 3}-1\right)^{2}
$$

In addition, we can approximate the condensation rate by the collision rate of monomers with the cluster

$$
v_{c}=\bar{c} \pi\left(r_{0}+r_{n}\right)^{2} \rho
$$

The average thermal velocity of the monomers in the gas is $\bar{c}=\left(8 k_{B} T_{g} / \pi m\right), \rho$ is the monomer density, $\mathrm{k}_{\mathrm{B}}$ is Boltzmann's constant, $\mathrm{T}_{\mathrm{g}}$ is the gas temperature and $m$ is the molecular mass, $\mathrm{r}_{0}$ and $r_{n}$ are the hard sphere equivalent radius of a monomer and a cluster. In this expression for the condensation rate, we approximate the cross section by the surface area of a sphere of radius $r_{n}$ and assume unit sticking probability upon collision. The hard sphere equivalent radius is calculated based on the gyration radius of the cluster $r_{\text {gyr }}$ (Arunachalam et al., 1999).

$$
r_{n}=\sqrt{\frac{5}{2}} r_{\text {gyr }}+r_{0}
$$

To compare the calculation results with the RRK theory, we calculate the evaporation and condensation rates for a set of simulation parameters (cluster size and temperature and gas density and temperature and interatomic potentials) that corresponds to the typical laser applications, such as laser ablation experiments (Handschuh et al., 1999; Vitiello et al; 2005).

\subsection{Combined MD-DSMC numerical model}

A combined MD-DSMC model is developed for the calculation of the laser plume formation and long-term evolution. In the model, two different numerical methods are used (i) molecular dynamics for target material disintegration and ejection of a mixture of clusters and monomers (14); and (ii) direct simulation Monte Carlo for the calculation of large-scale three-dimensional plume evolution (Itina et al., 1997). Here, as in the previous MD calculations (Zhigilei et al., 1998; Zeifman et al., 2002), the breathing sphere model is adopted to model laser pulse absorption and relaxation processes in the MD part. The calculation parameters in the MD part are the same as in these calculations for a molecular solid target. The pulse duration was set to be 15 ps.

The transition between the DSMC and MD calculations is performed by using MD results obtained at $1 \mathrm{~ns}$ after the laser pulse for the design of the initial conditions for the subsequent DSMC calculation. The collision probabilities in DSMC calculations are parameterized to describe the material properties in a gas phase, such as viscosity coefficient and velocity dependence of the equivalent collision cross sections. 
To couple the two models, all plume species are divided into five groups depending on their size. The groups are chosen as follows: 1 - monomers, 2 - clusters of 2 to 15 molecules, 3 clusters of 16 to 100 molecules, 4 - clusters of 101 to 1000 molecules, and 5 - clusters of more than 1000 molecules. The calculations are performed with laser pulse duration of $15 \mathrm{ps}$ and an absorbed laser fluence of $61.38 \mathrm{~J} / \mathrm{m}^{2}$. Cluster size is sampled in each group according to the size distributions obtained in the MD simulation.

In the present study, we assume that the spatial laser beam profile is top hat and the radial expansion of the ablation plume is small during the first MD stage. Therefore, the radial distributions of the simulation parameters are assumed to be uniform within the laser spot. The axial distributions of the initial DSMC parameters are determined by fitting the axial distributions of density, velocity, radial and axial temperatures for different groups obtained from the MD calculations. To fit the axial density distributions, we use normal distribution functions

$$
f_{i}(z)=\frac{d_{i}}{\sigma_{i} \sqrt{2 \pi}} \exp \left[-\left(z-\mu_{i}\right)^{2} / 2 \sigma_{i}^{2}\right]
$$

where $\mathrm{z}$ is the distance from the initial target surface. The parameters $\mathrm{d}_{\mathrm{i}}, \mu_{i}$, and $\sigma_{i}$ are calculated from numerical fits to the MD results performed for each group, and $i=1-5$. The linear dependencies

$$
g_{i}(z)=A_{i} z-B_{i}
$$

are used to fit axial velocity distributions, where $A_{i}$ and $B_{i}$ are fitting parameters for each component (Zhigilei, 2003). The distributions of the axial and radial temperatures as well as the internal temperatures of the clusters are fitted by six-order polynomial dependencies. More details of the procedure used for the statistical description of the output of MD simulations will be presented elsewhere.

\subsection{Modification of the DSMC method used for the calculation of ablation flows with clusters}

In the DSMC simulations, the laser plume is modeled by an ensemble of simulated particles (here, about $8 \cdot 10^{6}$ ). The simulated particles are introduced based on the gas-dynamic parameters obtained from the MD simulations of the initial stage of the plume formation. The physical space is divided into a network of cells with dimensions smaller than the local mean free path. The time is incremented by a small time step $\Delta t$. The calculations consist in the repetition of three main steps: (i) indexing of the simulated particles; (ii) calculation of a representative set of collisions and chemical reactions in each cell; (ii) movement of the simulated particles and calculation of the interactions with the boundaries (Bird, 1994). In the present work, collisions are calculated for all particles together based on their probabilities by using a NTC technique (Bird, 1994).

The initial and boundary conditions are set as follows. At the beginning, simulated particles are introduced according to the distributions calculated by MD as described in Section 2.2 above. The axis $r=0$ is set to be the axis of radial symmetry. At the target surface $(Z=0)$, a partial diffuse reflection and re-deposition of particles is sampled by using an accommodation coefficient. The accommodation coefficient of the target surface is set to be 
0.5 in this work. The simulated volume is increased several times during the calculation to cover the plume expansion region.

The calculation of collisions and reactions in the present DSMC includes the treatment of the following processes

i. elastic collisions;

ii. inelastic non-reactive collisions;

iii. sticking reactions in cluster - molecule collisions;

iv. aggregation reactions in cluster - cluster collisions;

v. cluster evaporation reactions.

The non-reactive collisions are calculated based on the conservation laws of energy and momentum. For clusters, the reference cross-sections are computed based on their effective radii $r_{n}=a n^{1 / 3}+b$, where $a=3.97 \AA, b=1.59 \AA, n$ is the number of monomers in the cluster. A soft-spheres collision model is used in the present modeling calculation. As we described above, the initial values of the internal energy, $E_{n}$, are assigned to each cluster based on the internal temperature distribution obtained from the MD simulation and the number of degree freedom in the cluster. To describe the internal energy modification in clusters and small molecules, Larsen-Borgnakke statistical model (Bird, 1994) is used. Sticking process is included for collisions in which the relative translational energy before the collision is smaller than the binding energy of the final cluster. In this case, the sticking of two clusters containing $n_{1}$ and $n_{2}$ molecules occurs with the probability (Hittema \& McFeaters, 1996)

$$
p_{1}=p_{0} \times\left(n_{1} n_{2}\right)^{-x}
$$

where $x$ is a parameter $\left(x=1 / 12\right.$ in the present simulation (33)), and $p_{0}$ is a constant. The parameters in (3) are selected based on a separate MD study of cluster evolution in a gas (to be published in a forthcoming paper). The binding energy in each cluster is given by a combination of volume and surface terms

$$
E_{b}=a_{V} n-a_{s} n^{2 / 3}
$$

where $a_{V}$ and $a_{S}$ are material constants and $n$ is the number of molecules in the cluster. To ensure the energy conservation in the sticking reaction, the relative translational energy of the monomer and cluster was transferred into the internal energy of the resulting cluster, whereas the center-of-mass velocity was conserved.

The rate of evaporation from a cluster of $n$ atoms with internal energy $E_{n}$ is calculated according to a simple exponential decay (Brady et al., 1979)

$$
\ln N / N_{0}=-k_{e v} t,
$$

where $k_{e v}$ is the decay constant, or the rate coefficient (Jarold, 1994).

\section{Results and discussion}

\subsection{Cluster evolution}

A series of calculation are performed for a cluster with different initial size, but with the same initial cluster temperature and gas density and temperature. Calculation results demonstrate that both evaporation [Fig. 1(a)] and condensation [Fig. 1(b)] rates increase with initial cluster 
size. The size dependencies are fitted by a polynomial function with the same order of the size function in theoretical rates. We obtain a reasonable agreement between the theoretical fits and our results. The evaporation and condensation rates are larger than $1.210^{11} \mathrm{~s}^{-1}$ for clusters containing around 500 atoms and larger than $2.010^{11} \mathrm{~s}^{-1}$ for $\mathrm{N}>1200$ atoms.

Furthermore, the initial cluster temperature is varied, keeping other initial parameters equal to those in the previous series of calculations. Simulations results demonstrate that the evaporation rate (Fig. 2(a)) decreases exponentially with the inverse of cluster temperature, while the cluster temperature dependency of the condensation rate is not significant (Fig.2(b)). In addition, one can see that the calculated dependency of evaporation rate can be described by the RRK theory. In the RRK theory, the evaporation rate exponentially decreases with cluster temperature and the coefficient in the exponential (sublimation energy) corresponds to the minimal kinetic energy required to obtain a significant evaporation. Our results show that we can determine the sublimation energy $D_{N}$ for the clusters. For instance, for nickel cluster with 604 atoms, we found $D_{N}=1.725 \mathrm{eV}$.
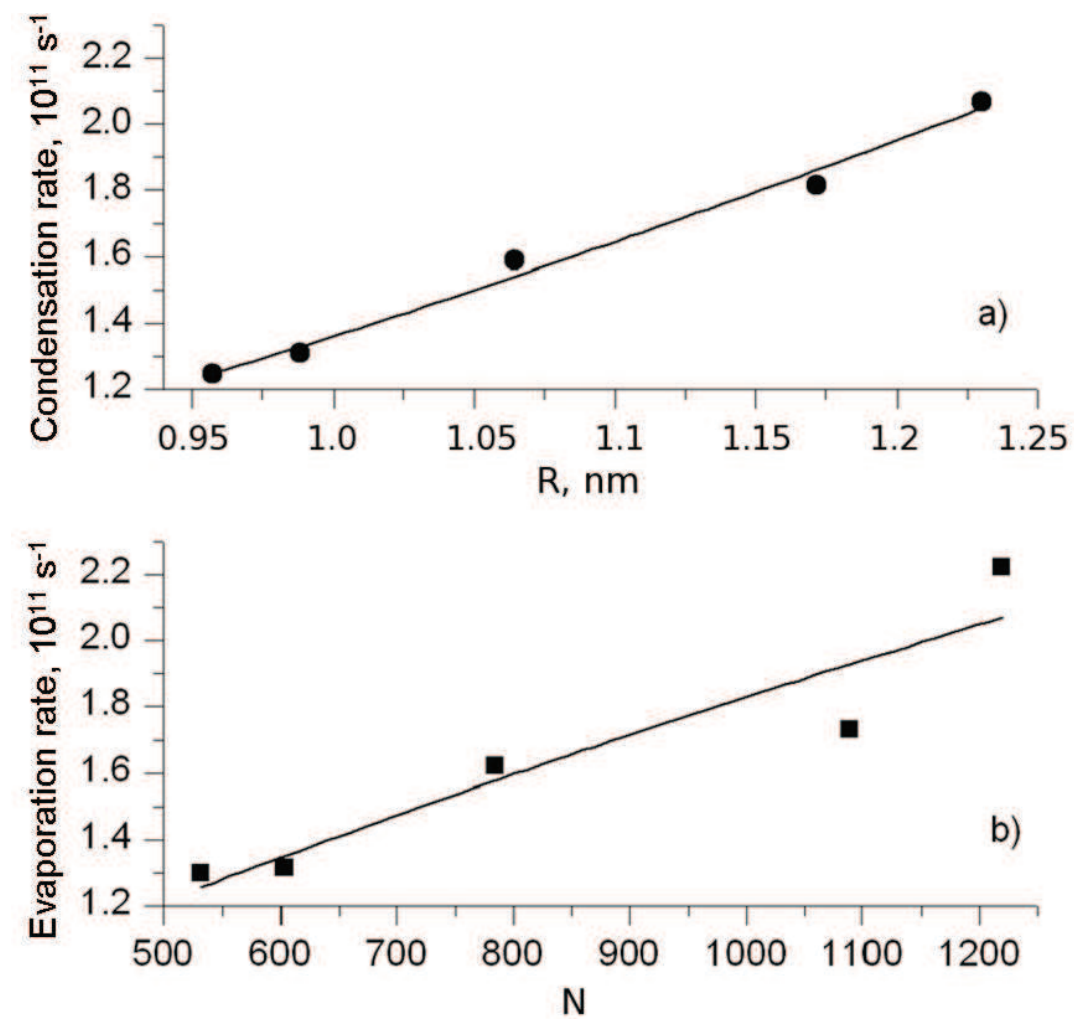

Fig. 1. (a) Condensation rate as a function of $\mathrm{R}$ cluster radius of nickel. The condensation rate is fitted by a function given by the collision rate such as $A+B\left(r_{1}+R\right)^{2}$, where $A=-1.973$ $1010 \mathrm{~s}^{-1}$ and $\mathrm{B}=1.18110^{29} \mathrm{~s}^{-1} \mathrm{~m}^{-2}$. (b) Evaporation rate as a function of the number of atoms, $\mathrm{N}$, in a nickel cluster. The evaporation rate is fitted by a function given by the RRK evaporation rate. These results are obtained for clusters of different sizes heated initially to $2400 \mathrm{~K}$ in a gas with the number density of $10^{19} \mathrm{~cm}^{-3}$ at $5036 \mathrm{~K}$. 

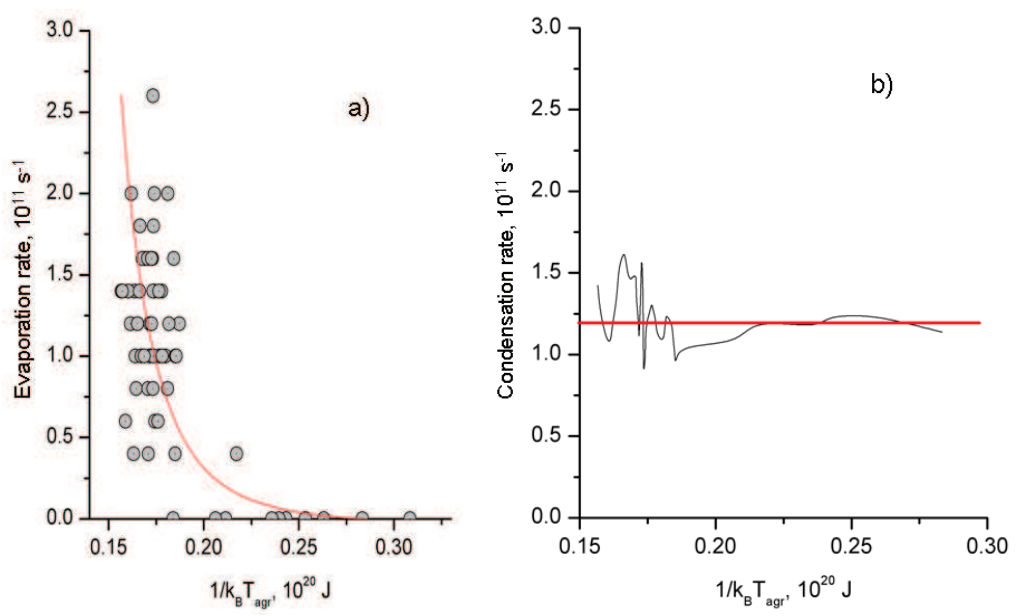

Fig. 2. (a) Evaporation rate as a function of cluster temperature of nickel. The evaporation rate is fitted by a function given by the RRK evaporation rate such as $A \exp \left(-D_{N} / k_{B} T\right)$, where $A=2.5710^{12} \mathrm{~s}^{-1}$ and $D_{N}=1.725 \mathrm{eV}$. (b) Condensation rate as a function of cluster temperature (nickel). The results are obtained for the cluster containing initially 604 molecules with different temperatures in a background gas with number density of $10^{19} \mathrm{~cm}^{-3}$ at $5036 \mathrm{~K}$.

Then, cluster evaporation and condensation processes are investigated by running our MD simulations with the same cluster parameters (material, initial size and temperature) but with different gas properties (density and temperature). Interestingly, the obtained calculation results show that the evaporation and condensation rates are proportional to the gas density (Fig. 3) and the square of temperature (Fig. 4). In particular, our simulations indicate a dramatic influence of both gas density and temperature on the evaporation process. For a nickel cluster with initially 604 atoms, calculations show that the evaporation rate is larger than $1.010^{11} \mathrm{~s}^{-1}$, if the initial gas temperature is larger than the boiling point ( $\mathrm{T}_{\text {boil }}=3190 \mathrm{~K}$ at $\mathrm{P}=0 \mathrm{~Pa}$, for nickel) and its density is larger than $1.010^{19} \mathrm{~cm}^{-3}$. So, under the given conditions, for both gas temperature and density, the evaporation process definitely prevails over the condensation one. Interestingly, an intersection is obtained at $4000 \mathrm{~K}$. This effect can be explained by interplay between the condensation and evaporation processes. At these temperatures, each process balances the other process, and the cluster size does not change significantly.

One can therefore expect that small clusters $(\mathrm{N}<50$ molecules) evaporate rapidly in the ablated plume regions where the density and the temperature of monomers are large $\left(T>T_{\text {boil }}\right.$ and $n>1.010^{19} \mathrm{~cm}^{-3}$ ). These conditions typically correspond to the beginning of the laser plume formation and its expansion in laser ablation.

Thus, the calculation results reveal the role of gas-cluster collisions in both evaporation and condensation processes. The performed MD simulations confirm that the RRK theory and the collision rate can be used to define the evaporation and condensation rates. Similar results for both studied materials are obtained. However, we can modify the RRK solution for the definition of evaporation and calculate the sticking probability for the condensation rate estimation based on our MD simulations. For this we propose analytical expressions for the evaporation $v_{e}$ and condensation $v_{c}$ rates, for large clusters of nickel and molecular material as follows 


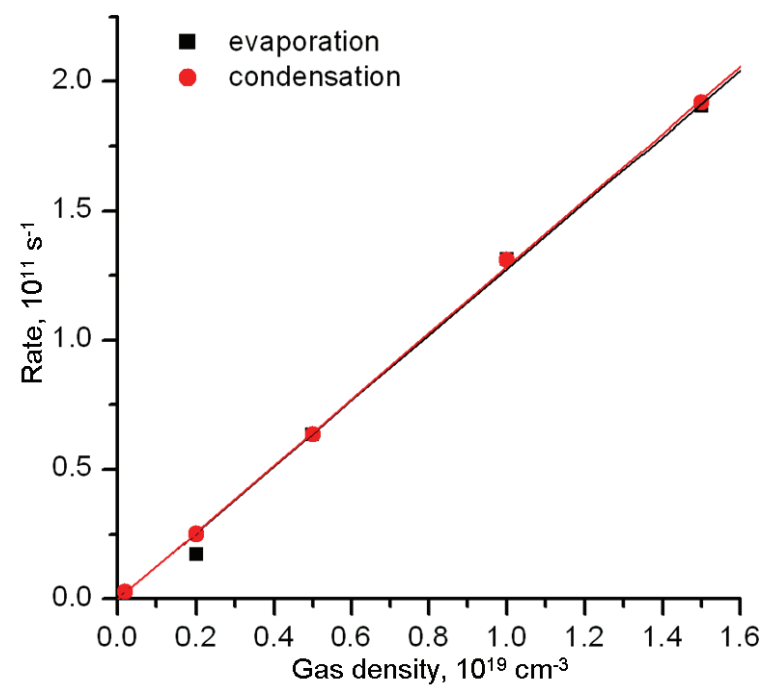

Fig. 3. Evaporation rates and condensation rates for a nickel cluster containing initially 604 molecules at $2490 \mathrm{~K}$ as a function of monomers gas density in a gas at $5000 \mathrm{~K}$. Linear fits are added to the dependencies.

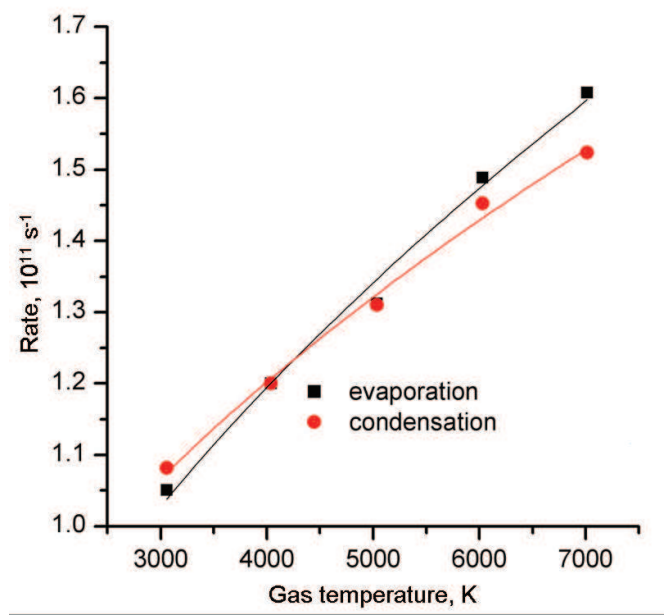

Fig. 4. Evaporation rates and condensation rates for a nickel cluster containing initially 604 molecules at $2490 \mathrm{~K}$ as a function of gas temperature. Here, the background gas number density is $10^{19} \mathrm{~cm}^{-3}$. 


$$
\begin{gathered}
v_{e}=v_{e}^{0} \bar{c} \rho g_{n} \exp \left[-\frac{D_{n}}{E_{n}}\right] \\
v_{c}=v_{c}^{0} \bar{c} \rho\left(r_{n}+r_{0}\right)^{2}
\end{gathered}
$$

where $v_{e}^{0}=9.1272 \cdot 10^{-18}$ and $v_{c}^{0}=5.4201$, the average thermal velocity of the monomers in the gas $\bar{c}=\left(8 k_{B} T_{g} / \pi m\right), \rho$ is the monomer density, $k_{B}$ is Boltzmann's constant, $T_{g}$ is the gas temperature and $m$ the molecular mass, $r_{0}$ and $r_{n}$ the hard sphere equivalent radius of a monomer and a cluster. $D_{n}$ is the molecular evaporation energy and $E_{n}$ is the internal energy.

\subsection{Plume dynamics and composition}

A series of calculations are performed with the combined MD-DSMC model. First, we consider the laser plume dynamics.

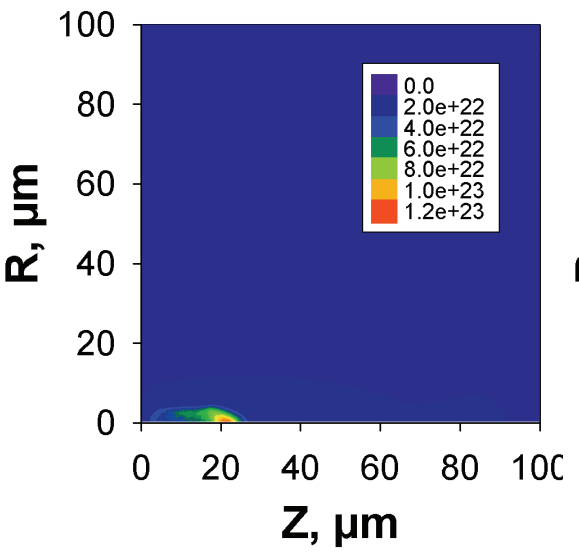

(a)

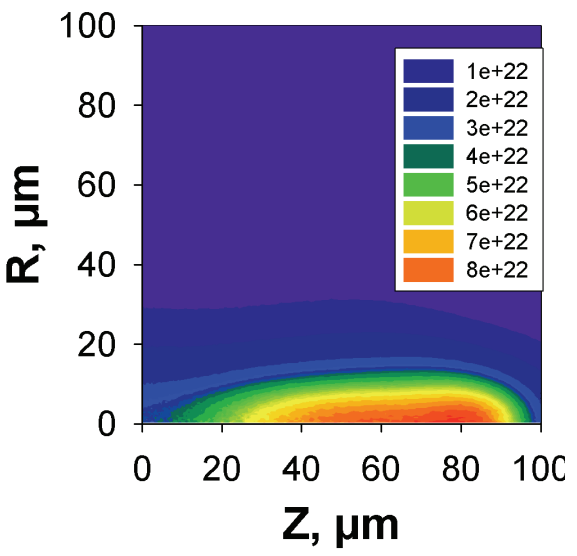

(b)

Fig. 5. Two-dimensional distributions of (a) cluster number density and (b) monomer density at a time delay of $50 \mathrm{~ns}$. The results are obtained in a MD-DSMC simulation performed for a pulse duration of $15 \mathrm{ps}$, laser fluence of $61 \mathrm{~J} / \mathrm{m}^{2}$, and a laser spot radius of $10 \mu \mathrm{m}$.

Distributions of the cluster and monomer densities shown in Fig. 5 illustrate the laser plume expansion away from the irradiated target. Monomers constitute the fastest plume component, whereas clusters are moving slower. In addition, we find that the larger are clusters the lower is their average axial velocity. Quantitatively, the center-of mass velocity of the monomer component of the plume is about $1560 \mathrm{~m} / \mathrm{s}$, whereas the one of clusters is as small as $555 \mathrm{~m} / \mathrm{s}$. Here, clusters bigger than 10 molecules were considered.

Furthermore, the changes in the plume composition and the influence of evaporation/condensation processes are examined. In order to provide a reliable description of the evaporation/condensation processes in DSMC, the cluster evolution is first investigated in a separate $5 \mathrm{~ns}$ long MD simulation performed for a gas box containing 
one cluster of a given initial size and temperature. These calculations demonstrate that, for temperatures observed by the end of MD simulations of laser ablation, small cluster evaporation prevailed over growth within the considered time of $5 \mathrm{~ns}$. The parameters used in the DSMC part are adjusted to these results.

The evolution of the plume composition in the MD-DSMC simulation of plume expansion is shown in Fig. 6 (a). One can see that at the beginning and until about $10 \mathrm{~ns}$, the number of clusters decreases, whereas the number of monomers rises. After that, the situation changes and the number of clusters in the plume steadily increases. At a delay of $200 \mathrm{~ns}$, the number of clusters reaches the one that was in the plume at $1 \mathrm{~ns}$ after the beginning of laser pulse. If one looks at the cluster abundance distribution [Fig. 6 (b)], which also varies with time, one can see that the number of small clusters decreases, while bigger clusters become more abundant.

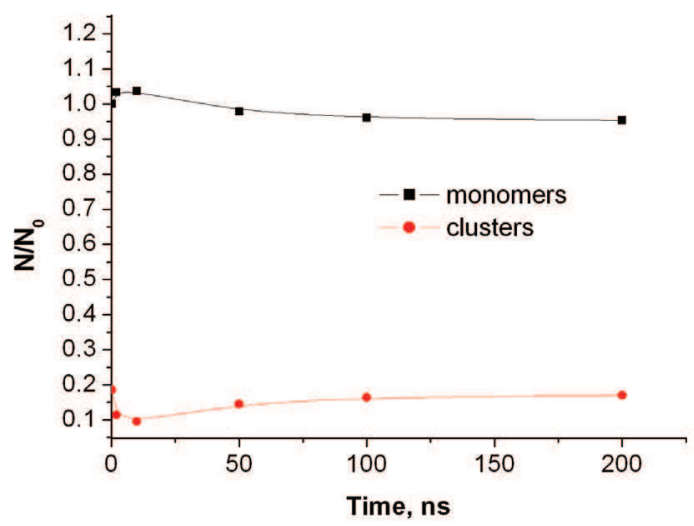

(a)

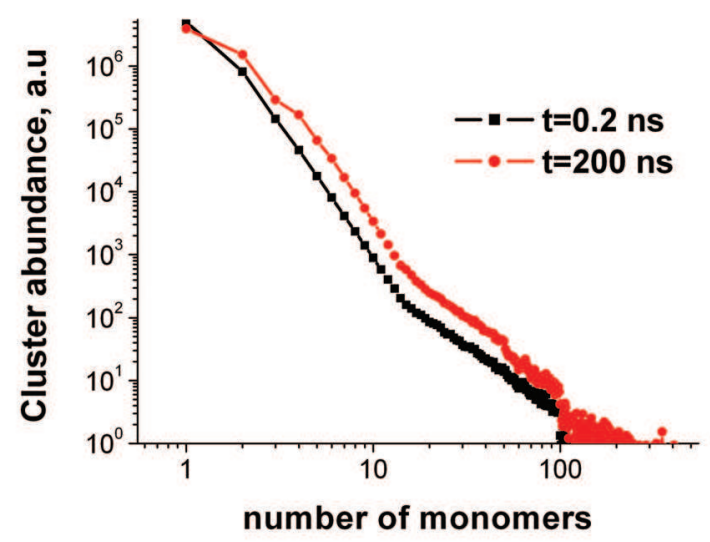

(b)

Fig. 6. (a) Time evolution of the number of monomers and clusters in the plume. (b) Cluster abundance distribution. The results are obtained in a MD-DSMC simulation performed for a pulse duration of $15 \mathrm{ps}$, laser fluence of $61 \mathrm{~J} / \mathrm{m}^{2}$ and a laser spot radius of $10 \mu \mathrm{m}$. 
As we have already seen from the MD calculations, the cluster size distribution obtained soon after the ejection is a decaying function. During the following plume expansion, numerous collisions take place in the plume and affect the distribution. The indication of these changes obtained in the MD-DSMC is displayed in Fig. 6(b). One can see the decrease in the number of small clusters and the growth in the number of average ones. The distribution shape agrees with the experimental findings. These results will be discussed in more details elsewhere.

The calculations may help explaining the results of the experiments on plume imaging and spectroscopy obtained for short and ultra-short laser pulses. Results of Noël et al (Noël and Hermann, 2009) show typical plume images recorded in our laboratory at different times in ultra-short laser ablation experiments with pulse width of $100 \mathrm{fs}$ at $400 \mathrm{~nm}$ wavelength. One can clearly observe two different plumes: the faster one and the slower one. Based on our simulation results and on additional spectroscopic study, we can conclude that the first component is composed of atoms and small clusters and the slower one is composed of bigger clusters.

\section{Conclusion}

We have studied the evaporation and condensation processes of a molecular cluster in the presence of a background gas composed of the same molecules as the cluster for two different materials: (i) molecular material, which is modeled by the breathing sphere model; and (ii) metallic material (nickel), which is modeled by the embedded atom model. We have analyzed the calculated evaporation rate by comparing the MD calculation results with the rates given by the RRK theory. The condensation rate is analyzed by using collision rate. As a result, we have observed that both gas density and temperature have strong influence on the evaporation process. We have shown that, in the presence of a gas, evaporation is a collision-driven process, just like condensation is. Therefore, the evaporation rate depends on gas density and temperature. As a result, both evaporation and condensation rates depend on the gas parameters.

The obtained MD results are in agreement with a slightly modified RRK collision rate expression. The corresponding modifications are proposed based on the MD calculation results. In particular, our calculation results have demonstrated that gas-cluster collisions affect cluster evaporation. The condensation rate has been also explained by using the cluster-gas collision rate with a sticking probability determined by our MD simulations.

Both the calculations performed with the combined MD-DSMC model and the experiments clearly demonstrate the role of two following channels of the cluster production by shortpulsed laser ablation

i. direct cluster ejection upon the laser-material interaction;

ii. collision-affected condensation and evaporation in the ablated plume flow.

The first process results from the fast energy absorption by the target, which leads to the explosive decomposition of a substantial volume of the rapidly heated target material. For short laser pulses, material decomposition might occur through either "phase-explosion" (Garrison et al., 2003), photomechanical spallation (Zhigilei \& Garrison 2000), or fragmentation (Perez \& Lewis, 2002; Glover, 2003) mechanisms. These processes take place in volume rather than on the surface and lead to the ejection of a mixture of clusters and monomers. 
The second mechanism is due to gas-phase collisions and evaporations. These processes are similar to the phenomena taking places in aggregation sources (Briehl \& Urbassek, 1999; Haberland, 1994). The major advantage of short and ultra-short laser pulses for cluster synthesis is the presence of the laser-ejected small molecules and clusters in the ablated flow. As a result, the formation of diatomic molecules in three-body collisions, which represents a "bottleneck" for cluster formation in common aggregation sources, is not crucial for cluster synthesis by short laser ablation.

\section{References}

Albert, O.; Roger, S.; Glinec, Y.; Loulergue, J. C.; Etchepare, J.; Boulmer-Leborgne, C.; Perriere, J. \& Millon, E. (2003). Appl. Phys. A: Mater Sci. Process, 76, 319

Amoruso, S. ; Bruzzese, R. ; Spinelli, N. ; Velotta, R. ; Vitello, M. \& Wang, X. (2004). Europhys. Lett. 67, 404

Amoruso, S.; Ausaniob, G. ; Bruzzese, R. ; Campana, C. \& Wang, X (2007). Appl. Surf. Sci., 254(4), p.1012

Anisimov, S. I. (1968). Zh. Eksp. Teor. Fiz. 54, 339 (Sov. Phys. JETP, 27, 182 (1968))

Arunachalam, V. ; Lucchese, R. R. \& Marlaow, W. H. (1999). Phy. Rev. E 60, p 2051

Bird, G. A. (1994). Molecular Gas Dynamics and the Direct Simulation of Gas Flows, Clarendon, Oxford.

Boldarev, A. S. ; Gasilov, V. A. ; Blasco, F. ; Stenz, C.; Dorchies, F.; Salin, F.; Faenov, A. Ya.; Pikuz, T. A.; Magunov, A. I. \& Skobelev, I. Yu (2001). JETP Letters, 73, 514

Brady, J. W.; Doll, J. D. \& Thompson, D. L. (1979). J. Chem. Phys., 71, 2467

Briehl, B. \& Urbassek, H. M. (1999). J. Vac. Sci. Technol. A 17, 256

Bulgakov, A. V. ; Ozerov, I.; Marine, W. (2004). Appl. Phys. A 79, 1591

Daw M. S. \& Baskes, M. I (1984). Phys. Rev. B, 29, p. 6443

Frenkel, D. \& Smit, B. (1996). Understanding molecular simulation, Academic Press

Garrison, B. J.; Itina, T. E. \& Zhigilei, L. V. (2003). Phys. Rev. E,68, 041501

Geohegan, D. B. ; Puretzky, A. A. ; Dusher, G. \& Pennycook, S. J. (1998). Appl. Phys. Lett., 72, 2987

Glover, T. E. (2003). J. Opt. Soc. Am. B, 20, 125

Gusarov, A. V. ; Gnedovets, A. V. \& Smurov, I. (2000). J. Appl. Phys. 88, 4362

Haberland, H. (1994). Clusters of Atoms and Molecules, ed. by H. Haberland (Springer, Berlin), p. 205

Handschuh, M. ; Nettesheim, S. \& Zenobi, R. (1999). Appl. Surf. Sci., 137(1-4) p. 125-135

Hittema H. \& McFeaters, J. S. (1996). J. Chem. Phys. 105, 2816

Itina, T. E.; Tokarev, V. N.; Marine, W. \& Autric, M. (1997). J. Chem. Phys. 106, 8905

Itina, T.E.; Hermann, J. ; Delaporte, P. \& Sentis, M. (2002). Phys. Rev. E, 66, 066406

Jarold, M. F. (1994). Clusters of Atoms and Molecules, ed. by H. Haberland (Springer, Berlin,), p. 163.

Kinjo, T.; Ohguchi, K. ; Yasuoka, K. \& Matsumoto, M. (1999). Computational Materials Science, 14, 138-141

Luk'yanchuk, B. ; Marine, W. \& Anisimov, S. (1998). Laser Phys. 8, 291

Makimura, T. ; Kunii, Y. \& Murakami, K. (1996). Jpn. J. Appl. Phys. Part 1, 35, 4780

Malakhovskii, A. V. \& Ben-Zion, M. (2001). Chem. Phys, 264, 135-143

Mizuseki, H. ; Jin, Y. ; Kawazoe, Y. \& Wille, L. T. (2001). Appl. Phys. A 73, 731 
Movtchan, I. ; Dreyfus, R. W. ; Marine, W. ; Sentis, M. ; Autric, M. \& Le Lay, G. (1995). Thin Solid Films 255, 286

Noël, S.; Hermann, J. (2009)Applied Physics Letters, 94:053120

Ohkubo, T.; Kuwata, M. ; Luk'yanchuk, B. ; Yabe, T. (2003). Appl. Phys. A., 77, 271

Perez, D. \& Lewis, L. J. (2002). Phys. Rev. Lett., 89, 25504

Schenter, G. K.; Kathmann, S. M. \& Garett, B. C. (1999). Phys. Rev. Letters, 82, 3484-3487

Vitiello, M.; Amoruso, S.; Altucci, C. ; de Lisio, C. \& Wang, X. (2005).Appl. Surf. Sci., 248(1-4), p. 404

Yamada, Y.; Orii, T.; Umezu, I. ; Takeyama, S. \& Yoshida, Y. (1996). Jpn. J. Appl. Phys. Part 1, 35,1361

Zeldovich, Y. B. \& Raizer, Yu. P. (1966). Physics of Shock Waves and High Temperature Hydrodynamic Phenomena Academic Press, London

Zeifman, M. I. ; Garrison, B. J. \& Zhigilei, L. V. (2002). J. Appl. Phys. 92, 2181

Zhigilei, L. V. ; Kodali, P. B. S. \& Garrison, B. J. (1998). J. Phys. Chem. B, 102, 2845-2853

Zhigilei, L. V. \& Garrison, B. J. (2000). J. Appl. Phys. 88, (3), 1281

Zhigilei, L. V. (2003). Appl. Phys. A 76, 339

Zhong, J. ; Zeifman, M. I. \& Levin, D. A. (2006). J. Thermophysics and Heat Transfer, 20, 41-45 


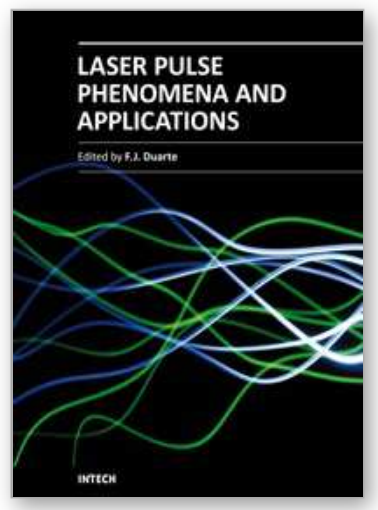

\author{
Laser Pulse Phenomena and Applications \\ Edited by Dr. F. J. Duarte
}

ISBN 978-953-307-405-4

Hard cover, 474 pages

Publisher InTech

Published online 30, November, 2010

Published in print edition November, 2010

Pulsed lasers are available in the gas, liquid, and the solid state. These lasers are also enormously versatile in their output characteristics yielding emission from very large energy pulses to very high peak-power pulses.

Pulsed lasers are equally versatile in their spectral characteristics. This volume includes an impressive array of current research on pulsed laser phenomena and applications. Laser Pulse Phenomena and Applications covers a wide range of topics from laser powered orbital launchers, and laser rocket engines, to laser-matter interactions, detector and sensor laser technology, laser ablation, and biological applications.

\title{
How to reference
}

In order to correctly reference this scholarly work, feel free to copy and paste the following:

Tatiana Itina (2010). Mechanisms of Nanoparticle Formation by Laser Ablation, Laser Pulse Phenomena and Applications, Dr. F. J. Duarte (Ed.), ISBN: 978-953-307-405-4, InTech, Available from:

http://www.intechopen.com/books/laser-pulse-phenomena-and-applications/mechanisms-of-nanoparticleformation-by-laser-ablation

\section{INTECH}

open science | open minds

\section{InTech Europe}

University Campus STeP Ri

Slavka Krautzeka 83/A

51000 Rijeka, Croatia

Phone: +385 (51) 770447

Fax: +385 (51) 686166

www.intechopen.com

\section{InTech China}

Unit 405, Office Block, Hotel Equatorial Shanghai

No.65, Yan An Road (West), Shanghai, 200040, China

中国上海市延安西路65号上海国际贵都大饭店办公楼 405 单元

Phone: +86-21-62489820

Fax: +86-21-62489821 
(C) 2010 The Author(s). Licensee IntechOpen. This chapter is distributed under the terms of the Creative Commons Attribution-NonCommercialShareAlike-3.0 License, which permits use, distribution and reproduction for non-commercial purposes, provided the original is properly cited and derivative works building on this content are distributed under the same license. 